\title{
Lower Gray Matter Density in the Anterior Cingulate Cortex and Putamen Can Be Traceable in Chronic Heroin Dependents After Over Three Months of Successful Abstinence
}

\author{
Ahmadreza Keihani, ${ }^{1}$ Hamed Ekhtiari ${ }^{1,2}$ Seyed Amir Hossein Batouli, ${ }^{1}$ Alireza Shahbabaie, ${ }^{1}$ Nahid \\ Sadighi, ${ }^{3}$ Mahdieh Mirmohammad, ${ }^{1}$ and Mohammad Ali Oghabian ${ }^{1, *}$ \\ ${ }^{1}$ Neuroimaging and Analysis Group, Research Center for Molecular and Cellular Imaging, Tehran University of Medical Sciences, Tehran, Iran \\ ${ }^{2}$ Neurocognitive Laboratory, Iranian National Center for Addiction Studies, Tehran University of Medical Sciences, Tehran, Iran \\ ${ }^{3}$ Advanced Diagnostic and Interventional Radiology Research Center (ADIR), Tehran University of Medical Sciences, Tehran, Iran \\ "Corresponding author: Mohammad Ali Oghabian, Neuroimaging and Analysis Group, Research Center for Molecular and Cellular Imaging, Tehran University of Medical \\ Sciences, Tehran, Iran. Tel: +98-9121962851, Fax: +98-66581533, E-mail: oghabian@tums.ac.ir
}

Received 2016 August 28; Revised 2016 October 19; Accepted 2017 January 25.

\begin{abstract}
Background: In recent years, a few studies have addressed the effect of chronic heroin use on brain structure with respect to volume and shape; however, the literature in this field is sparse and further studies are necessary to generate robust replications.

Objectives: In this study, we intended to assess gray matter density (GMD) differences between successfully abstinent heroin dependents and healthy subjects. Indirect evidences for the causal role of drug use in the GMD differences are also targeted here by testing the hypothesis on the correlation between GMD differences and duration of drug use.

Patients and Methods: Using structural magnetic resonance imaging (MRI) and voxel-based morphology (VBM) technique, changes in gray matter volume and density were evaluated in 18 abstinent heroin dependents and 20 age-, education- and gendermatched healthy subjects. Heroin dependents were all male, and at least 3 months successfully abstinent (mean abstinence duration $=9.2 \pm 5.2$ months).

Results: Decreased GMD in the right anterior cingulate cortex and bilateral putamen were found in abstinent heroin dependents in comparison to controls, corrected for multiple comparisons $(\mathrm{P}<0.05)$. Also, partial correlation analysis, corrected for age, showed negative correlations between total intracranial volume (TIV) and total GMD ( $r=-0.61$ and -0.44 , respectively) with the duration of opiate usage in heroin dependents. GMD in the right putamen also had negative correlation with the duration of drug abuse $(r=$ -0.49) and a weak negative correlation was observed between left putamen density and duration of drug abuse $(r=-0.42)$. Examination of the negative correlation between the duration of drug abuse before the age of 25 years and total GMD, while controlling for age, showed weak negative results $(r=-0.44)$.

Conclusion: Specific structural changes were noticeable in prefrontal and striatal areas in the heroin dependents even after a period of successful abstinence. Our results on the interaction between duration of drug use and some of these structural changes provides preliminary ideas for reciprocal cause and effect roles for brain structural changes and heroin abuse. These findings might also imply that brain structures are unable to return to normal state and cannot recover spontaneously even after months of abstinence. This evidence reminds us of the importance of neurorehabilitation interventions after termination of drug use.
\end{abstract}

Keywords: Addiction, Heroin, Structural Magnetic Resonance Imaging (sMRI), Gray Matter Density (GMD), Voxel-Basedmorphometry (VBM)

\section{Background}

United Nations estimates up to 16.5 million illicit opiate users worldwide, up to three quarters of which use heroin. Europe and Asia remain the key global consuming markets (1).

In fact, opiate usage, especially heroin, remains a great health and social problem around the world. Brain imaging has undergone marked development in recent years with the advent of dedicated imaging techniques and modalities to help in better understanding the mecha- nisms of addiction, and to assess changes in the central nervous system (CNS) in different types of addiction (2-7). Yet, only limited studies have focused on brain changes among heroin dependents (6, 8-14). These evaluations have revealed both anatomical (grey matter and white matter) and functional changes among heroin addicts. Anatomically, brain structural assessments have shown grey matter (GM) volume reduction mainly in prefrontal, frontal, temporal and cingulate cortices $(9,15-17)$. In addition, some cognitive impairments and behavioral abnormalities have 
been shown among heroin addicts, including higher impulsiveness during problem solving, increased commission errors during response suppression tasks, working memory impairments and abnormality in goal-directed behavior $(6,18,19)$. Similar to other types of addiction, considering these two parallel findings in neuroimaging and cognitive functions, researchers have tried to find correspondence between them for more insight into heroin addiction mechanisms and its corresponding brain changes. It has been shown that drugs could have direct effect on brain tissue (20), which could be site-specific for each drug. These changes could be responsible for behavioral changes and cognitive impairments in the patients. Moreover, some primary and inherent brain abnormalities could be considered as predisposing factors for addiction. Understanding these corresponding changes is important in determining more exact mechanisms of the disease, which seems necessary for devising more effective prevention strategies and improved treatment planning. In assessing the natural course of brain changes among heroin addicts, both disease progression and disease recovery (i.e. during abstinence period) are important. In fact, comparison of the brain structural changes in the normal subjects with abstinent subjects and active abusers could better elucidate the nature and natural course of the brain changes and psychiatric abnormalities among heroin addicts. Most papers published in this field have focused on brain structural changes in active users $(9,16)$ and very limited papers have assessed the brain findings among 4.9, 3 months and 3 years abstinent heroin dependents respectively $(15,17,21)$.

\section{Objectives}

The current study therefore aims at comparing brain imaging results in a group of abstinent heroin dependents with healthy controls to assess gray matter density (GMD) differences between the two groups. We will also examine the causal role of drug use in the GMD differences by testing the hypothesis on the correlation between GMD differences and duration of drug use.

\section{Patients and Methods}

\subsection{Participants}

Twenty heroin-dependent and 22 healthy control righthanded subjects were recruited in this study. Subjects did not have a history of neurologic disorders and none of the subjects reported head trauma. All abstinent heroin subjects were the members of "Congress 60 " with documents for at least 3 months of opiate abstinence confirmed with drug urinalysis (UT). Congress 60 is an organization that manages networks of centers that treat abuse of abstinentbased substances in Iran. All heroin-dependent subjects met diagnostic and statistical manual of mental disorders (DSM) IV-TR (22) criteria for heroin dependence (before participating in the treatment program). None of the heroin dependent and healthy control subjects had any major psychiatric or neurologic disorders except drug abuse disorder. All participants had negative urine tests for opiate and stimulant drugs at least 90 days before the scanning session based on their treatment center report (according to the center's policy, urine check was carried out twice per week) and were rechecked during the study at the time before image acquisition for each subject. All subjects were male and Persian-speakers. Using the Mann-Whitney Utest, there were no significant differences in the age $(\mathrm{P}$ value $=0.17)$ and education $(P$ value $=0.08)$ among subjects (Table 1).

Table 1. Demographics of Normal Controls and Abstinent Heroin Dependents and Characteristics of Drug Abuse Profile in the Abstinent Heroin Dependents ${ }^{\mathrm{a}}$

\begin{tabular}{|lcc|}
\hline Characteristics & Heroin Abstinent Group & Control Group \\
\hline Sample size & 18 & 20 \\
\hline Gender (M, F) & $\mathrm{M}$ & $\mathrm{M}$ \\
\hline Age, $\mathbf{y}$ & $29.8 \pm 4.65$ & $26.90 \pm 3.5$ \\
\hline \multicolumn{1}{|c}{ Range } & $22-36$ & $18-43$ \\
\hline Education, $\mathbf{y}$ & $11.2 \pm 1.35$ & $13.27 \pm 2.16$ \\
\hline $\begin{array}{l}\text { Duration of drug abuse, } \mathbf{y} \\
\text { Abstinence periods, mo }\end{array}$ & $13.8 \pm 5.3$ & $\mathrm{~N} / \mathrm{A}$ \\
\hline $\begin{array}{l}\text { Duration of drug abuse } \\
\text { before age of 25, } \mathbf{y}\end{array}$ & $9.2 \pm 5.6$ & $\mathrm{~N} / \mathrm{A}$ \\
\hline $\begin{array}{l}\text { Duration of opiate abuse, } \\
\text { mo }\end{array}$ & $8.3 \pm 4.2$ & $\mathrm{~N} / \mathrm{A}$ \\
\hline
\end{tabular}

Abbreviations: F, female; M, male; N/A, not applicable; y, year; mo, month. ${ }^{\mathrm{a}}$ Values are expressed as mean \pm SD.

\subsection{Image Acquisition}

Structural images were acquired on Avento $1.5 \mathrm{~T}$ scanner (Siemens, Erlangen, Germany). Three-dimensional T1weighted images were obtained with magnetization prepared rapid gradient echo (MPRAGE) protocol, echo time $(\mathrm{TE})=3.55 \mathrm{~ms}$, repetition time $(\mathrm{TR})=1910 \mathrm{~ms}$, voxel size 1 $\times 1 \times 1 \mathrm{~mm}^{3}$, and flip angle $=30$ degrees. At the end, each subject had $192 \times 256 \times 176$ structural image matrix size. Voxel-based morphometry (VBM) analysis, described in the next section, was implemented on the structural images.

\subsection{Preprocessing}

First of all, digital imaging and communications in medicine (DICOM) images were converted to neuroimag- 
ing informatics technology initiative (NIFTI) format using the dcm2niigui software package, and then the matrix size and orientation of all data were visually checked using MRIcro. Quality control was implemented in MATLAB (Release 2013b, The MathWorks, Inc., Natick, Massachusetts, United States). Signal to noise ratio (SNR) and homogeneity of images were checked in the quality control step, and consequently two heroin dependents and two control subjects were excluded from the analysis due to their low quality MRI data. All data were aligned and saved with the same orientation before the VBM analysis.

\subsection{Voxel-Based Morphometry}

VBM analysis was performed using SPM8 (Welcome Department of Imaging Neuroscience: http://www.fil.ion.ucl.ac.uk/spm), including the following steps: 1) segmenting the T1-weighted images into grey matter (GM) and white matter (WM) tissue types, using the "new segment" toolbox; 2) generation of the native space as well as Diffeomorphic Anatomical Registration Through Exponentiated Lie Algebra (DARTEL)-imported versions of the segmented tissue types; 3) populationspecific templates were generated by iteratively averaging the DARTEL-imported data of the GM and WM tissue types, using "Create Template" toolbox of the SPM8; 4) this template, as well as the flow field data generated during the template construction were used to normalize each individual's GM and WM data to the standard montreal neurological institute (MNI) space (Montreal Neurological Institute, Canada). This resulted in images with the matrix size of $145 \times 121 \times 121$, and a cubic voxel with the dimension of $1.5 \mathrm{~mm}^{3}$. Finally, images were smoothed with a $10 \mathrm{~mm}$ full-width half-maximum (FWHM) Gaussian kernel before any further analysis.

Total brain GM and WM volumes of the subjects of the two groups were initially estimated, by adding the probability estimates of the voxels of each map, and then multiplying the resulted value to $3.375 \mathrm{~mm}^{3}$ (the volume of one voxel). Total intracranial volume (TIV) was also estimated for both groups. Using global calculation, voxel-wise comparison of the two groups was also performed in SPM8, using independent two-tailed two-sample t-test analysis. The significance level was set at the corrected P value $<0.05$ (corrected for multiple comparisons using family wise error correction). To eliminate any effect of edge difference between the two images, a relative threshold was selected for implicit masking of the final images.

\subsection{Ethics}

The written consent form was approved by the Independent ethics committee of Tehran University of Medical
Sciences and was signed by the subjects after a full explanation of the study procedure.

\subsection{Statistical Analysis}

We used SPSS 20 (IBM Corp. Released 2011. IBMSPSS Statistics for Windows, Version 20.0. Armonk, NY: IBM Corp.) and SPM8 (http://www.fil.ion.ucl.ac.uk/spm) for performing statistical analysis. Details of each analysis step was described above in related sections.

\section{Results}

For the heroin-dependent group, the average duration of heroin and other drug substance usage was $13.8 \pm 5.3$ years. Drug in our study included opium, puppy milk, heroin, crack, norjizak, tamjizak, ecstasy, cannabis, and sedatives. The relationship between duration of drug usage and whole-brain GM volume was assessed using SPSS.

\subsection{VBM Analysis Results}

Reduced GM volume in three brain clusters was demonstrated by the VBM technique in heroin dependents compared with healthy subjects, including right anterior cingulate cortex and bilateral putamen (corrected P value $<0.05$ ) (Figure 1 and Table 2).

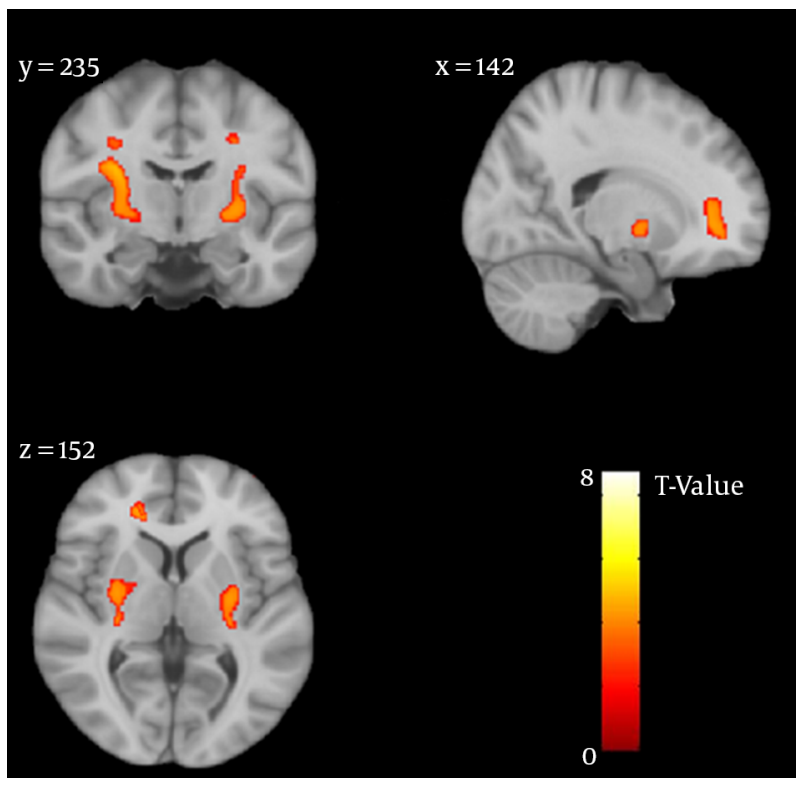

Figure 1. Representative slices in brain map (Standard Space: avgMNI152), showing regions of reduced gray matter density in the heroin-abstinence group compared with control group. 
Table 2. Clusters with Reduced GM Density in Heroin Abstinence Group vs. Healthy Subjects

\begin{tabular}{|c|c|c|c|c|c|c|c|}
\hline \multirow[t]{2}{*}{ Anatomical Region } & \multirow[t]{2}{*}{ Right/Left } & \multicolumn{3}{|c|}{ MNI Coordinate } & \multirow[t]{2}{*}{ Cluster Size (> 100) (No of Voxels) } & \multirow[t]{2}{*}{ Peak T-Value } & \multirow[t]{2}{*}{ Cluster P Value (Corr) } \\
\hline & & $\mathbf{x}$ & $\mathbf{y}$ & $\mathbf{z}$ & & & \\
\hline Putamen & $\mathrm{R}$ & 26 & -6 & 2 & 974 & 7.63 & 0.001 \\
\hline Putamen & $\mathrm{L}$ & -26 & -6 & 2 & 593 & 7.49 & 0.003 \\
\hline Anterior Cingulate & $\mathrm{R}$ & 19.5 & 36 & 5 & 239 & 7.34 & 0.007 \\
\hline
\end{tabular}

Abbreviation: GM, grey matter; MNI, montreal neurological institute.

\subsection{Correlation Analysis Results}

Partial Spearman rank order correlation was used to control for possible confounding effect of age. Partial correlation analysis results showed that at $\mathrm{P}$ value $<0.05$, duration of the drug abuse had a negative correlation with abstinent heroin-dependent's TIV $(r=-0.48)$. Also, we observed significant negative correlations between GM density and duration of drug abuse $(r=-0.42)$ in the same group.

Duration of drug abuse had significant correlations with VBM results of the right putamen $(r=-0.49)$ and negative correlation by the left putamen $(r=-0.42)$ density, but no correlation was found between duration of addiction and anterior cingulate cortex (ACC) density.

We defined an arbitrary cut-off-point for brain maturation (i.e. 25 years old) to obtain the duration of drug abuse during this critical time of development and investigate the correlation of this variable with the GMD in the abstinent heroin dependents. Negative correlation was obtained between the duration of drug abuse before the age of 25 and GMD while controlling age $(r=-0.44)$.

\section{Discussion}

Using advanced MRI imaging techniques, many researches have tried to assess the structural and functional brain alterations in drug-dependent individuals. Neuroimaging studies on heroin abusers have frequently addressed functional differences $(9,17,21,23)$. Although there are few studies on brain structure differences of heroin abusers, their results are controversial.

\subsection{Interpretation and Comparison of Results}

The present study found that abstinent heroin abusers had reduced GM density in three regions: right anterior cingulate cortex and bilateral putamen. These results are consistent with a number of investigations that reported brain tissue impairment in some specific regions in the opiate-dependents $(9,16,17,24)$. Each study, however, emphasizes on a different series of cortical or subcortical clusters. Among the various brain regions, cingulate cortex (especially the anterior division) is a common area that is found to be reduced in size among heroin dependents in comparison with the control healthy group. Yuan et al. (15) displayed decreases in GM density in the prefrontal cortex, anterior cingulate cortex (ACC), and insular and temporal cortices on 30 lifetime heroin dependents with the average abstinence duration of 5 months. Using VBM analysis, reduced GM density were found in the frontal cortex, cingulate cortex, and the occipital regions in heroin dependents after three days of abstinence (17). No significant difference was found in the superior frontal gyrus between heroin addicts and the control group when heroin dependents had undergone an abstinence period of one month. Structural impairments were however observed in the right middle frontal gyrus, left cingulate gyrus, and left inferior occipital gyrus (25). Cingulate cortex is specialized in cognitive control by activating goal-directed behavior and modifying cognitive processes in order to obtain internal aims (16). The current study found decreased density of ACC in heroin addicts even after months of abstinence in contrast with the matched controls. Although there was a negative correlation between duration of addiction and bilateral putamen density, such correlation was not significant with the density of ACC. On the other hand, limited structural studies on the brains of heroin dependents have displayed inconsistent results, containing various affected brain regions due to opiate usage. In a recent study (21), reduced brain tissue was observed in the surrounding of the parieto-occipital sulcus, which included the precuneus and cuneus in former heroin-dependent subjects which were abstinent for a number of years. Moreover, Liu et al. reported gray matter density decrease in the right prefrontal cortex, left supplementary motor cortex, and bilateral cingulate cortices in heroin abusers (16). In our investigation, a decreased bilateral putamen was observed contradictory to a study carried out by Franklin et al. (25) that showed increased bilateral putamen density in nicotine users. In addition, another study documented higher density of the right ventral anterior putamen in amphetamine type stimulant and cocaine users compared with healthy subjects (26). Putamen is found to be involved 
in a variety of cognitive functions such as motor execution and movement control. The density of putamen has negative correlation with impulsivity in the subjects with attention deficit hyperactivity disorder (ADHD) (27), and positive correlation with compulsive behaviors in obsessive compulsive disorder (OCD) subjects (28). As drug abuse is contained of both impulsive and compulsive components, the status of left and right putamen should be investigated closely.

As the destructive effect of drug usage on cortical and subcortical anatomy is a complex multivariate issue, a wide range of differences among findings in the neuroimaging studies could be caused by multiple variables such as abstinence duration, different treatment profiles, and age of drug onset. One other reason explaining the inconsistency between findings of different studies is the substantial comorbidity related to other psychoactive drug usages and different addiction and abstinence conditions among different studies $(9,17,29)$. Negative correlation between brain volumes and duration of heroin abuse is replicated in VBM studies (9). In the present study, we examined total intracranial volumes (TIV) related to length of opiate abuse. Our results demonstrated TIV decrease with increasing years of opiate abuse. The study results also showed abnormality in heroin-dependent individuals' GMD in some regions of the brain volume (i.e. right ACC and bilateral putamen, as mentioned above) compared with normal healthy subjects. These findings are in agreement with some other studies (30). We also addressed the effect of drug abuse duration on the brain before the age of 25 to evaluate the consequences of heroin abuse on the dependents' brain before cortical maturation. Based on longitudinal studies, the prefrontal regions of the brain are exposed to anatomical changes until the early 20s (31-33). Drug usage onset before the structural and functional brain maturation might cause more severe alterations in gray matter and cortical structures. Therefore, the age of 25 was defined as a cut-of-point below which the duration of abuse was calculated for the addicts. For an age threshold of 25, results showed negative correlation between heroin abusers' GMD and onset of abuse duration when the effect of age was kept constant.

\subsection{Limitations}

There are several limitations in our study that must be considered in interpreting the findings. Some gray matter abnormality in abstinent duration of this study is not consistent with other studies, suggesting that the change in GMD in heroin dependents after short period of abstinence may be subtle, and possibly the small sample size in this study was not sufficient to detect these subtle changes. We compared the two groups while their smoking status could not be fully matched; nicotine effect is difficult to determine separately. We cannot specifically determine the effects of tobacco smoking on the structural aspects of the brain because of the overlapping of other drug abuse and tobacco abuse; thus the effect of smoking must be considered in interpreting our results. The results here are gender-based because all subjects in this study were male, and therefore, the results cannot be generalized to females.

\subsection{Conclusion}

We conclude that specific structural changes were noticeable in prefrontal and striatal areas in the heroin dependents even after a period of successful abstinence. Interaction between duration of drug use and some of these structural changes provides preliminary ideas for reciprocal cause and effect roles for brain structural changes and heroin dependence. Our results also imply that brain structures are unable to return to normal state and cannot recover spontaneously even after months of abstinence; therefore, the evidences ahead remind us of the importance of neurorehabilitation interventions after termination of drug use.

\section{Acknowledgments}

We thank Mitra Ebrahimpour for review of our statistical analysis method.

\section{Footnotes}

Authors' Contributions: Study content and question were designed by Mohammad Ali Oghabian, Hamed Ekhtiari, Sayed Amir Hossein Batouli, and Ahmadreza Keihani. Data Acquisition and experimental setup were performed by Nahid Sedighi. Data analysis and statistical analysis was done by Ahmadreza Keihani, Hamed Ekhtiari, Sayed Amir Hossein Batouli, and Alireza Shahbabaei. Draft of manuscript was written by Ahmadreza Keihani, Mahdieh Mirmohammad, Hamed Ekhtiari, and Seyed Amir Hossein Batouli. Analytical and critical edit were carried out by Hamed Ekhtiari, Sayed Amir Hossein Batouli, and Ahmadreza Keihani. Final acute revision was performed by Hamed Ekhtiari, Sayed Amir Hossein Batouli, and Mohammad Ali Oghabian.

Financial Disclosure: None declared.

Funding/Support: None declared. 


\section{References}

1. UNODC . World drug report. ; 2013.

2. Kuhn S, Schubert F, Gallinat J. Reduced thickness of medial orbitofrontal cortex in smokers. Biol Psychiatry. 2010;68(11):1061-5. doi: 10.1016/j.biopsych.2010.08.004. [PubMed: 20875635].

3. Thompson PM, Hayashi KM, Simon SL, Geaga JA, Hong MS, Sui Y, et al. Structural abnormalities in the brains of human subjects who use methamphetamine. J Neurosci. 2004;24(26):6028-36. doi: 10.1523/JNEUROSCI.0713-04.2004. [PubMed: 15229250].

4. Lorenzetti V, Batalla A, Cousijn J. Cannabis Use Disorders and Altered Brain Morphology: Where Is the Evidence? Curr Addict Rep. 2016;3(2):189-98. doi: 10.1007/s40429-016-0102-2.

5. Koenders L, Cousijn J, Vingerhoets WA, van den Brink W, Wiers RW, Meijer CJ, et al. Grey Matter Changes Associated with Heavy Cannabis Use: A Longitudinal sMRI Study. PLoS One. 2016;11(5):e0152482. doi: 10.1371/journal.pone.0152482. [PubMed: 27224247].

6. Mwansisya TE, Zhang H, Wang Z, Wu G, Hu A, Wang P. Major Depressive Disorder and Heroin-dependent Patients Share Decreased Frontal Gray Matter Volumes [U+202F]: A Voxel-Based Morphometry Study. ; 2016.

7. Zhong J, Shi H, Shen Y, Dai Z, Zhu Y, Ma H, et al. Voxelwise meta-analysis of gray matter anomalies in chronic cigarette smokers. Behav Brain Res. 2016;311:39-45. doi: 10.1016/j.bbr.2016.05.016. [PubMed: 27173432].

8. Forman SD, Dougherty GG, Casey BJ, Siegle GJ, Braver TS, Barch DM, et al. Opiate addicts lack error-dependent activation of rostral anterior cingulate. Biol Psychiatry. 2004;55(5):531-7. doi: 10.1016/j.biopsych.2003.09.011. [PubMed: 15023582].

9. Yuan Y, Zhu Z, Shi J, Zou Z, Yuan F, Liu Y, et al. Gray matter density negatively correlates with duration of heroin use in young lifetime heroin-dependent individuals. Brain Cogn. 2009;71(3):223-8. doi: 10.1016/j.bandc.2009.08.014. [PubMed: 19775795].

10. Liu H, Li L, Hao Y, Cao D, Xu L, Rohrbaugh R, et al. Disrupted white matter integrity in heroin dependence: a controlled study utilizing diffusion tensor imaging. Am J Drug Alcohol Abuse. 2008;34(5):562-75. doi: 10.1080/00952990802295238. [PubMed: 18720268].

11. Reid a G., Daglish M. R., Kempton M. J., Williams T. M., Watson B., Nutt D. J. . Reduced thalamic grey matter volume in opioid dependence is influenced by degree of alcohol use: a voxel-based morphometry study. J Psychopharmacol. 2008;22(1):7-10.

12. Xiao Z, Lee T, Zhang JX, Wu Q, Wu R, Weng X, et al. Thirsty heroin addicts show different fMRI activations when exposed to water-related and drug-related cues. Drug Alcohol Depend. 2006;83(2):157-62. doi: 10.1016/j.drugalcdep.2005.11.012. [PubMed: 16406379].

13. Fu LP, Bi GH, Zou ZT, Wang Y, Ye EM, Ma L, et al. Impaired response inhibition function in abstinent heroin dependents: an fMRI study. Neurosci Lett. 2008;438(3):322-6. doi: 10.1016/j.neulet.2008.04.033. [PubMed: 18485592].

14. HE Z, ZHOU XH, WANG XY. Longitudinal Observation on Brain Structure in the Heroin Dependence Patients During Abstince. Chinese J Clin Psychol. 2008;4:009.

15. Yuan K, Qin W, Dong M, Liu J, Sun J, Liu P, et al. Gray matter deficits and resting-state abnormalities in abstinent heroin-dependent individuals. Neurosci Lett. 2010;482(2):101-5. doi: 10.1016/j.neulet.2010.07.005. [PubMed: 20621162].

16. Liu H, Hao Y, Kaneko Y, Ouyang X, Zhang Y, Xu L, et al. Frontal and cingulate gray matter volume reduction in heroin dependence: optimized voxel-based morphometry. Psychiatry Clin Neurosci. 2009;63(4):563-8. doi:10.1111/j.1440-1819.2009.01989.x. [PubMed: 19531112].

17. Wang X, Li B, Zhou X, Liao Y, Tang J, Liu T, et al. Changes in brain gray matter in abstinent heroin addicts. Drug Alcohol Depend. 2012;126(3):304-8. doi: 10.1016/j.drugalcdep.2012.05.030. [PubMed: 22717339].
18. Nielsen DA, Ho A, Bahl A, Varma P, Kellogg S, Borg L, et al. Former heroin addicts with or without a history of cocaine dependence are more impulsive than controls. Drug Alcohol Depend. 2012;124(1-2):11320. doi: 10.1016/j.drugalcdep.2011.12.022. [PubMed: 22265192].

19. Motlagh F, Ibrahim F, Menke JM, Rashid R, Seghatoleslam T, Habil $\mathrm{H}$. Neuroelectrophysiological approaches in heroin addiction research: A review of literatures. J Neurosci Res. 2016;94(4):297-309. doi: 10.1002/jnr.23703. [PubMed: 26748947].

20. Bega DS, McDaniel LM, Jhaveri MD, Lee VH. Diffusion weighted imag ing in heroin-associated spongiform leukoencephalopathy. Neurocrit Care. 2009;10(3):352-4. doi: 10.1007/s12028-008-9172-x. [PubMed: 19116698].

21. Wang L, Zou F, Zhai T, Lei Y, Tan S, Jin X, et al. Abnormal gray matter volume and resting-state functional connectivity in former heroin-dependent individuals abstinent for multiple years. Addict Biol. 2016;21(3):646-56. doi: 10.1111/adb.12228. [PubMed: 25727574].

22. American Psychiatric Association (APA) . Diagnostic and statistical manual of mental disorders: DSM-IV-TR®. American Psychiatric Association; 2000.

23. Tabatabaei-Jafari H, Ekhtiari $\mathrm{H}$, Ganjgahi $\mathrm{H}$, Hassani-Abharian $\mathrm{P}$ Oghabian MA, Moradi A, et al. Patterns of brain activation during craving in heroin dependents successfully treated by methadone maintenance and abstinence-based treatments. JAddict Med. 2014;8(2):123-9. doi: 10.1097/ADM.0000000000000022. [PubMed: 24637623].

24. Weller RE, Stoeckel LE, Milby JB, Bolding M, Twieg DB, Knowlton RC et al. Smaller regional gray matter volume in homeless african american cocaine-dependent men: a preliminary report. Open Neuroimag J 2011;5:57-64. doi: 10.2174/1874440001105010057. [PubMed: 22135719].

25. Franklin TR, Wetherill RR, Jagannathan K, Johnson B, Mumma J, Hager $\mathrm{N}$, et al. The effects of chronic cigarette smoking on gray matter volume: influence of sex. PLoS One. 2014;9(8):ee104102. doi: 10.1371/journal.pone.0104102. [PubMed: 25090480].

26. Mackey S, Stewart JL, Connolly CG, Tapert SF, Paulus MP. A voxel-based morphometry study of young occasional users of amphetamine-type stimulants and cocaine. Drug Alcohol Depend. 2014;135:104-11. doi 10.1016/j.drugalcdep.2013.11.018. [PubMed: 24342420].

27. Gozzi A, Agosta F, Massi M, Ciccocioppo R, Bifone A. Reduced limbic metabolism and fronto-cortical volume in rats vulnerable to alcohol addiction. Neuroimage. 2013;69:112-9. doi: 10.1016/j.neuroimage.2012.12.015. [PubMed: 23261637].

28. Gillan CM, Robbins TW. Goal-directed learning and obsessivecompulsive disorder. Philos Trans R Soc Lond B Biol Sci. 2014;369(1655) doi: 10.1098/rstb.2013.0475. [PubMed: 25267818].

29. Ahmadi Soleimani SM, Ekhtiari H, Cadet JL. Drug-induced neurotoxicity in addiction medicine: From prevention to harm reduction. Prog Brain Res. 2016;223:19-41.

30. Lyoo IK, Pollack MH, Silveri MM, Ahn KH, Diaz CI, Hwang J, et al. Prefrontal and temporal gray matter density decreases in opiate dependence. Psychopharmacology (Berl). 2006;184(2):139-44. doi: 10.1007/s00213-005-0198-x. [PubMed: 16369836].

31. Lenroot RK, Giedd JN. Brain development in children and adolescents: insights from anatomical magnetic resonance imaging. Neurosci Biobehav Rev. 2006;30(6):718-29. doi: 10.1016/j.neubiorev.2006.06.001. [PubMed: 16887188].

32. Giedd JN, Blumenthal J, Jeffries NO, Castellanos FX, Liu H, Zijdenbos A, et al. Brain development during childhood and adolescence: a longitudinal MRI study. Nat Neurosci. 1999;2(10):861-3. doi: 10.1038/13158. [PubMed: 10491603].

33. Gogtay N, Giedd JN, Lusk L, Hayashi KM, Greenstein D, Vaituzis AC, et al. Dynamic mapping of human cortical development during childhood through early adulthood. Proc Natl Acad Sci U S A. 2004;101(21):8174-9. doi: 10.1073/pnas.0402680101. [PubMed: $15148381]$ 